\title{
A novel HACCP system supported by QMRA for increased food safety
}

\author{
D. Popov Janevska, R. Gospavic \& V. Popov \\ Wessex Institute of Technology, UK
}

\begin{abstract}
A novel approach based on integration of the existing Hazard Analysis and Critical Control Point (HACCP) approach with the Quantitative Microbial Risk Assessment (QMRA) is presented which is developed as part of the Chill-On EC FP6 research project. Though the paper deals with the main principles of operation of the system, some elements of practical implementation and operation of the HACCP system combined with QMRA as well as a Shelf Life Predictor (SLP) are presented. The novel HACCP system makes use of QMRA for estimating the risk to consumers due to consumption of food contaminated with Specific Foodborne Pathogens (SFP) or estimating the remaining shelf life of the product. The system is designed for help with increasing the quality and safety of products in food supply chains.
\end{abstract}

Keywords: food safety, HACCP, QMRA, shelf life.

\section{Introduction}

Microbiological hazards in food can normally be present in the raw food material or contamination can occur as a result of poor hygiene during processing from equipment, staff handling the food and temperature abuses. But even if bacteria is present in food, that does not mean their level is high enough to cause illness in people. They become dangerous when their number increases to a point where they can cause illness or produce toxins that are dangerous. To prevent this from happening conditions, which can lead to increase in bacterial counts, need to be kept under control. This can be done by combining HACCP and QMRA into a new system. This is why application of the QMRA in food supply chains for enhancing the safety of the food products is becoming more attractive [1]. 
In this work the use of predictive modelling for enhancing the performance of the HACCP systems is discussed. The improvement would bring increased safety of food products. Such system is developed as part of the Chill-On EC FP6 Programme and is based on a novel HACCP-QMRA approach. The system includes a SLP as well, though usually shelf life is not considered in HACCP. The QMRA and SLP can help to detect any changes in the supply chain which could have negative impact on the quality and safety of the product, before HACCP or supply chain operators can notice the changes, providing this way sufficient time for corrective actions. It is proposed that the system would make use of continuous monitoring of environmental parameters, e.g., temperature, $\mathrm{pH}$, as well as results of testing for SFP and Specific Spoilage Organisms (SSO). The system would use the Supply Chain (SC) data stored in the system database over a period of time in order to calculate periodically changes in model predictions in risk levels or shelf life. Application of appropriate statistical analysis would identify significant variations in the trends in terms of decreased safety or shelf life of the product which would require further attention and corrective actions. Such system can be applied for reducing the impact of seasonal variations in temperature on food quality and safety.

\section{Current regulations and HACCP}

Hazard Analysis and Critical Control Point (HACCP) concept was first developed in the 1960 s by NASA. It was designed to prevent microbial, physical and chemical hazards in food for the space missions. Today HACCP is an internationally recognized system (set of guidelines) and is widely used for safe food production. If properly applied it could help to achieve a higher level of food safety. For the system to work it needs to be applied by food companies along the food chain.

The new food law in EU, Regulation 178/2002 that applies from 1st January 2005 states that food companies have primary responsibility for safe food. From 1st January 2006 new food hygiene law Regulation 852/2004 requires all food companies in all member states (except farms) to operate food safety programmes based on the seven HACCP principles contained in the Codex Alimentarius.

It is probable that HACCP in the future will develop in such way to incorporate also the Quantitative Microbial Risk Assessment (QMRA). HACCP is a qualitative approach and it has been pointed out that qualitative risk assessment cannot address the process's inherent variability in any meaningful manner [2]. Since QMRA is a quantitative approach, a synthesis of both approaches should produce a food safety system which will be able to offer higher reliability. One of the aspects where QMRA can clearly help HACCP is that it can provide quantitative estimates of the risk in the critical control points taking into account the variability of the parameters in the food supply chain. It is also possible that HACCP integrated with QMRA will be easier to compare for equivalence worldwide, due to the added quantitative dimension. If the 
QMRA is implemented in practice, the determination of equivalence between very different systems should be more straightforward.

\section{Implementation of QMRA in supply chains}

The main purpose of the QMRA is to provide an estimate of the risk to consumers due to contamination of food with pathogen bacteria. The QMRA consists of the following elements (see Figure 1): (i) hazard identification, (ii) exposure assessment, (iii) hazard characterization, (iv) risk characterization.

\subsection{Hazard identification}

Hazard identification is the essential step in the QMRA where the specific foodborne pathogens (SFP) relevant to the considered supply chain are identified. This step is supply chain specific in terms of SFP that might be present or introduced at a certain point in the supply chain.

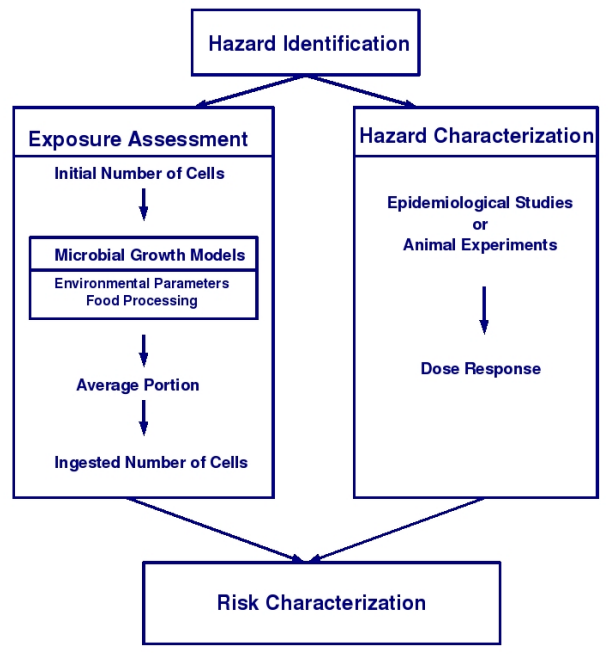

Figure 1: $\quad$ Elements of the QMRA.

\subsection{Exposure assessment}

The main purpose of exposure assessment is to establish the ingested number of cells of SFP by the consumer. A food supply chain can be very complex and can have many steps and processes.

Basic input and output variables in each step are the concentration of bacterial organisms $(\mathrm{N})$ and prevalence $(\mathrm{P})$. These quantities should be considered as uncertain variables in the model. The uncertainty in different environmental and supply chain parameters can be included into the mathematical model by using stochastic models, e.g., Monte Carlo simulation. 
In order to establish the probability density function for the ingested number of SFP, quantitative information on the incidence, prevalence and count of pathogens in the product are required. This information has to be collected through testing of the product over certain period of time, e.g., one year, in order to produce reliable data. The testing would also provide data to determine the probability for cross contamination in the SC.

\subsubsection{Microbial growth models}

The microbial growth models are used to predict the growth of microorganisms in the product under certain environmental conditions. During the development of the mathematical models for growth of particular microorganism the first step is to perform laboratory experiments in order to provide the necessary data. The second step is to prepare a supply chain specific microbial growth model. It is important to note that such models would be reliable as long as the conditions in the supply chain stay within the range of parameters used in the experiments.

\subsubsection{Primary models}

The primary models provide information on the growth of certain bacteria in constant environmental conditions $[3,4]$ and they are used in both, the QMRA and the SLP. These models are relevant only for storage at the specific constant environmental conditions. Since the temperature varies in supply chains the primary microbial growth models cannot cover the whole supply chain.

\subsubsection{Secondary models}

The secondary models $[5,6]$ are able to predict bacterial growth in a condition of variable environmental parameters, e.g., T, $\mathrm{pH}, \mathrm{NaCl} \%$, water activity $\left(a_{\mathrm{w}}\right)$, atmosphere. The main assumption is that the relative growth rate is changing instantly in dynamic environmental conditions. The most important parameter in the secondary models is usually the temperature.

The one of the most important model parameters, specific growth rate, is dependant on the environmental parameters and the commonly used secondary models for specific growth rate are: the model of Ratkowsky et al. [7], models based on $\gamma$ concept or complete model of Zwietering et al. [8], cardinal temperature model with inflection (CTMI) [9], cardinal temperature and $\mathrm{pH}$ model (CTPM) [10] and sub optimal models [11].

\subsubsection{Deterministic and stochastic models}

The microbial growth models can be deterministic or probabilistic/stochastic. The deterministic models do not take into account random distributions of the initial cell population as well as random behaviour of the bacterial organisms during the multiplication. In the contrast to the deterministic microbial growth models the stochastic ones can take into account the high level of variability and uncertainty in environmental and biological parameters and as outcome can produce the probability for microbial concentration at different time instants or at different points within the supply chain.

It is convenient to use the probability density function for finding certain number of SFP cells in the supply chain as an input parameter for the stochastic microbial growth models. 


\subsection{Hazard characterization}

An essential part of the QMRA is a suitable dose response model for estimating the probability for illness due to ingestion of certain number of SFP cells. However, the accurate dose-response relation is difficult to describe for two reasons: (1) the variability in both host susceptibility and microorganism infectivity; and (2) the lack of experimental data [12,13].

Several statistical models have been used to describe microbial dose response relation. The most often reported dose response models for SFP in the literature are the log-normal, log-logistic, simple exponential, flexible exponential, BetaPoisson, Weibull-Gamma and Gompertz [14, 15].

\subsection{Implementation of the SLP}

The implementation of the SLP model is simpler than the implementation of the QMRA model. The SLP model uses a microbial growth model similarly to the QMRA. Which SSO to be selected would be product and supply chain specific. The rejection point, in terms of microbial count, is determined by experiments involving sensory evaluation.

\section{QMRA and SLP implementation in a food supply chain}

The main benefit of the implementation of the QMRA and SLP modules in a supply chain is related to near-real-time data processing and provision of information to the supply chain operators on the risk levels to consumers and remaining shelf-life of the product taking into account variations in environmental parameters. Here it will be assumed that the technology which transfers automatically data from sensors in the supply chain to the QMRA and SLP modules exists. It will be considered that the QMRA and SLP modules are part of a Decision Support System (DSS). The frequency with which the data is transmitted to the DSS depends on the environmental parameters of interest.

Figure 2 shows a possible implementation of the QMRA module. The necessary Real Time Data (RTD) and input parameters for risk assessment analysis of microbial contamination along the supply chain is provided through communication with the DSS and from the database. The operation of the module depends on the supply chain specific data which enables preparation of primary and secondary models for SFP of interest. A dose response model for each SFP is also necessary together with the process analysis module. The QMRA itself is imagined as a generic software module and can operate for various food supply chains, providing that the database with the necessary information, e.g., for calibration of the microbial growth models and dose response model, can be produced. As can be seen in Figure 2 a possibility for update of the databases exists. Such updates are necessary in order to take into account changes in the supply chain, or in order to fine tune the models to the specific supply chain. The experiments for establishing the parameters of the microbial growth models are usually conducted in a laboratory environment. In 
the experiments if a product from the supply chain is used higher probability for agreement between the model predictions and actual microbial growth rates in the supply chain is expected. This however does not guarantee a perfect agreement between the model's predictions and the observations in the supply chain. Fine tuning of the models can be further carried out in the specific supply chain in order to achieve higher accuracy of the predictions. The above discussion is also valid for the SLP model.

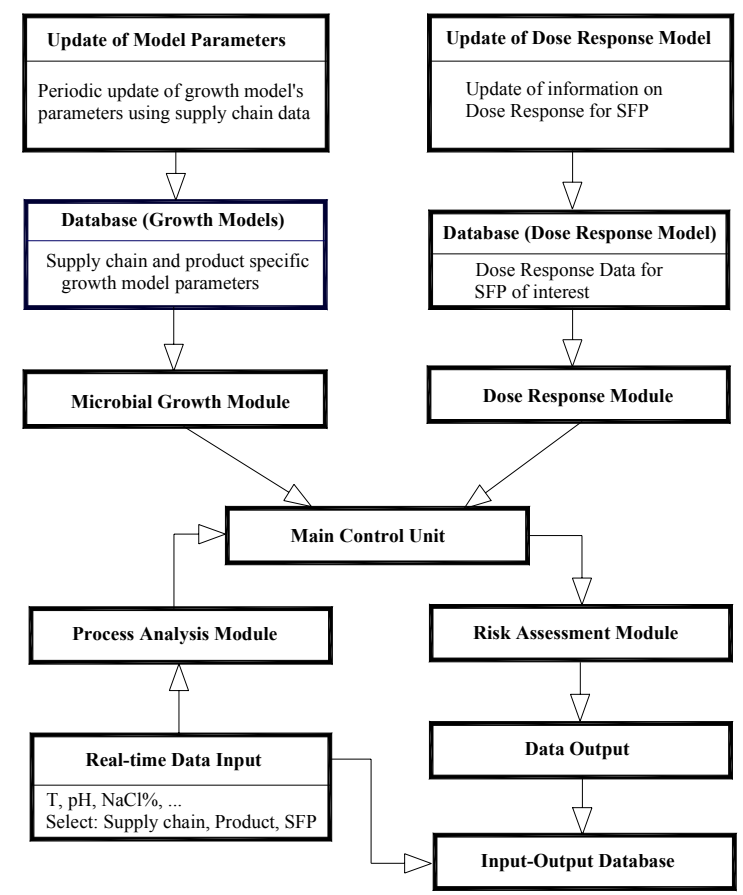

Figure 2: $\quad$ Block diagram of the QMRA module.

\subsection{Operation of the QMRA and SLP modules in real time}

An efficient supply of real time data along the supply chain is essential for successful operation of the DSS. It may occur that in some parts of the food supply chain such real time data supply is possible, and for these parts the data should be stored and sent to the DSS as soon as possible.

The environmental temperature as one of the most important parameters for predictive microbiology should be monitored in real time using thermometers with data loggers and where possible the data should be transmitted/sent to the DSS in real time. The simplified data transmission between the DSS and QMRA/SLP is presented in Figure 3.

The SLP can be implemented as deterministic or stochastic model while the QMRA should be implemented only as a stochastic model since it will provide a 


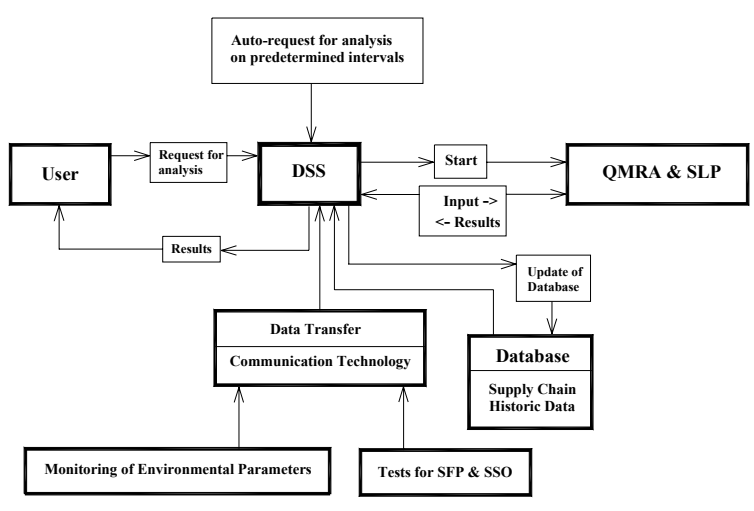

Figure 3: Block diagram of interaction of DSS and QMRA and data transfer.

probability for illness upon consumption of the product. The deterministic model uses less number of operations and therefore is faster however the utility of the stochastic model is higher.

\section{Novel approach integrating HACCP with QMRA}

There are several differences between the HACCP and the QMRA approach. The main differences are: (i) HACCP is qualitative analysis while QMRA is quantitative analysis; (ii) HACCP deals with a variety of hazards while QMRA considers SFPs only; (iii) HACCP is operated by people while QMRA module can operate independently of supply chain (SC) actors by receiving real time data from sensors and by performing analysis at predefined intervals. QMRA can also launch a warning to predefined set of SC operators, depending on the location of the product in the SC; (iv) HACCP defines critical control points (CCP) and limits that have to be preserved in order to keep risks of food poisoning within acceptable limits. QMRA can provide almost continuous information, while the product is at any point within the SC, on the risk of illness at the moment of consumption; (v) The DSS can incorporate the SLP module as well. Though the SLP is not related to the risks to consumers, HACCP procedures may be implemented in order to utilize both, the QMRA as well as the SLP module, with a notion that the SLP results are not utilized by the HACCP.

With the integration of the QMRA and SLP as parts of the DSS with the HACCP it is expected that the following improvements will be achieved: (i) The risk assessment provided by QRMA upgrades the HACCP decision with quantitative information; (ii) More accurate and nearly-continuous SC predictions on the risk levels for the consumers and remaining shelf life depending on the monitoring data in the SC; (iii) Warnings sent to SC operators independent of the HACCP and location of control points in the SC; (iv) The verification procedures of the HACCP for pathogen bacteria is helped by the QMRA; (v) Procedures (testing) implemented in HACCP to verify accuracy of 
the QMRA and SLP; (vi) The corrective actions for the HACCP can be added to the DSS, therefore reducing possibility for mistakes; (vii) Full compatibility of the QMRA-HACCP system with the HACCP procedures required in different countries can be achieved; (viii) Early detection of change in the SC which require corrective actions by periodical analysis of historic data using the QMRA and SLP models.

\section{Aspects of the practical operation of the system}

The concept is shown in Figure 4. The figure shows a simplified fish SC. It can be seen that the data is passed from the SC, ideally continually at selected intervals through the SC information system, to the DSS. The frequency with which environmental parameters are passed to the DSS depends on how rapidly these parameters can change. The temperature is the parameter which can change more rapidly than the other parameters and is also more important than the other parameters so it would be recorded with higher frequency.

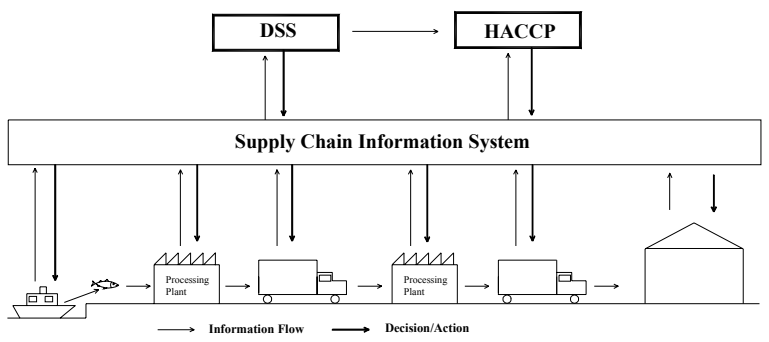

Figure 4: Supply chain analysis using DSS (QMRA \& SLP) - HACCP approach.

HACCP will be applied at each CCP. There will be different operators implementing the HACCP at different CCPs. Supply chain operators implementing HACCP will be able to receive the data from the SC directly at the point where they are and from the DSS for the whole SC. They will receive the results of analysis performed by the QMRA and will follow the procedures to preserve the safety of the product. The classical HACCP works with critical limits, but in this case there are also results of analysis which show in more accurate way the probability for certain risk level to the consumer at time of purchase/consumption of the product. The critical limits may not be sufficiently clear indicators of the safety of the product in certain point in the SC if the previous temperature in the SC up to that point is not analyzed. Therefore it may happen that the DSS shows that the risk is increased, based on all the available data at the DSS though the HACCP shows that everything is normal, and vice versa. The DSS may conclude that despite slight temperature abuse in certain part of the SC the product is still safe for consumption, though the HACCP will consider the product unsafe since the critical limits have been exceeded. Such 
conflicts would require further attention in order to be resolved. This would most likely involve more extensive testing than the routinely performed one in order to determine whether the product is safe. The data obtained may be used to adapt the HACCP which will resolve such conflicts in the future. Such system would be more reliable than a system which is based on a single approach, HACCP or QMRA. Since HACCP and QMRA are based on different principles, qualitative and quantitative, respectively, the probability that both systems fail (give wrong estimate) at same instance is largely reduced.

\section{Conclusions}

A new system is discussed which would utilize a novel HACCP-QMRA approach as well as a Shelf Life Prediction (SLP) model to secure safety and quality of food products. Various aspects of the implementation are discussed together with the benefits of such system for increasing the safety and quality of food products in supply chains. It is expected that the system will be able to provide early warning for changes in risk levels to consumers due to consumption of the product as well as shelf life of the product in the supply chain. The HACCP - QMRA\&SLP system is currently being implemented within the Chill-On FP6 EC funded project.

\section{Acknowledgement}

The financial support by the European Commission through the FP6 - Food Quality and Safety programme, Contract No. FOOD-CT-2004-513953, is gratefully acknowledged.

\section{References}

[1] McMeekin, T.A., Baranyi, J., Bowman, J., Dalgaard, P., Kirk, M., Ros, T., Schmid, S., Zwietering, M.H. (2006) Information systems in food safety management. International Journal of Food Microbiology 112, 181-194.

[2] Buchanan, R. L., \& Whiting, R. C. (1998). Risk assessment: a means for linking HACCP plans and public health. J. Food Protect., 61(11), 15311534.

[3] Baranyi J., Roberts T. A. (2006) Mathematics of predictive food microbiology, International Journal of Food Microbiology, 26, 199-218.

[4] Swinnen, I.A.M., Bernaerts, K., Dens, E.J.J., Geeraerd, A.H., Van Impe, J.F. (2004) Predictive modelling of the microbial lag phase: a review, International Journal of Food Microbiology, 94, 137-159.

[5] Baranyi J., Roberts T. A. (1994) A dynamic approach to predicting bacterial growth in food - Review Paper. International Journal of Food Microbiology, 23, 277-294.

[6] Gospavic R., Kreyenschmidt J., Bruckner S., Popov V., Haque N. (2008) Mathematical modelling for predicting the growth of Pseudomonas spp. in 
poultry under variable temperature conditions. International Journal of Food Microbiology, 127, 290-297.

[7] Ratkowsky, D.A., Olley, J., McMeekin, T.A. and Ball, A. (1982) Relationship between temperature and growth rate of bacterial cultures. $J$. Bacteriol. 149, 1-5.

[8] Zwietering, M.H., Wijtzes, T., De Wit, J. C., Van’t Riet, K. (1992) A decision support system for prediction of microbial spoilage in foods. $J$. Food Prot, 55, 973-979.

[9] Rosso, L., Lobry, J.R., and Flandrios, J.P. (1993) An unexpected correlation between cardinal temperatures of microbial growth highlighted by a new model, J. Theoretical Biology, 162, 447-463.

[10] Rosso, L., Lobry, J.R., Bajard, S., Flandrios, J.P. (1995) Convenient model to describe the combined effects of temperature and $\mathrm{pH}$ in microbial growth, Applied and environmental microbiology, 610-616.

[11] Wijtzes, T., McClure, P.J., Zwietering, M.H., Roberts, T.A. (1993) Modelling bacterial growth of Listeria monocytogenis as a function of water activity, $\mathrm{pH}$ and temperature, International Journal of Food Microbiology, 18, 139-149.

[12] Farber, J.M., Rose, W.H., Harwig, J. (1996) Health risk assessment of Listeria monocytogenes in Canada, Intl. J. Food Microbiol., 30, 145-154.

[13] Holcomb, D.L., Smith, M.A., Ware, G.O., Hung, Y.C., Brackett, R.E., Doyle, M.P. (1999) Comparison of six dose-response models for use with foodborne pathogen, Risk Anal. 19 (6), 1091-1100.

[14] Coleman, M., Marks, H. (1998) Topics in dos-response modelling. J. of Food Protection, 61(11), 1550-1559.

[15] Medema, G.J., Teunis, P.F.M., Havelaar, A.H., Haas, C.N. (1996) Assessment of the dose-response relationship of Campylobacter jejuni, Intl. J. Food Microbiol., 30, 101-111. 